Département de sciences économiques

9902

\title{
Coherent Cost-Sharing Rules
}

SPRUMONT, Yves 


\section{Département de sciences économiques}

Université de Montréal

Faculté des arts et des sciences

C.P. 6128, succursale Centre-Ville

Montréal (Québec) H3C 3J7

Canada

http://www.sceco.umontreal.ca

SCECO-information@UMontreal.CA

Téléphone : (514) 343-6539

Télécopieur : (514) 343-7221

Ce cahier a également été publié par le Centre interuniversitaire de recherche en économie quantitative (CIREQ) sous le numéro 0299.

This working paper was also published by the Center for Interuniversity Research in Quantitative Economics (CIREQ), under number 0299.

ISSN 0709-9231 


\title{
CAHIER 9902
}

\section{COHERENT COST-SHARING RULES}

\author{
Yves SPRUMONT ${ }^{1}$
}

1 Centre de recherche et développement en économique (C.R.D.E.) and Département de sciences économiques, Université de Montréal

February 1999

The author is grateful to E. Friedman, H. Moulin, and Y. Wang for insightful discussions, and to the Social Sciences and Humanities Research Council (SSHRC) of Canada for financial support. 


\title{
RÉSUMÉ
}

Nous réexaminons la version discrète du modèle axiomatique de partage de coûts. Nous proposons une condition de cohérence (informationnelle) qui exige que la solution d'un problème soit identique à celle donnée à au moins un de ses raffinements. Nous prouvons qu'une règle linéaire strictement cohérente doit être une règle d'ordre aléatoire simple.

Mots clés : partage de coûts, additivité, ordre aléatoire

\begin{abstract}
We reconsider the discrete version of the axiomatic cost-sharing model. We propose a condition of (informational) coherence requiring that not all informational refinements of a given problem be solved differently from the original problem. We prove that strictly coherent linear cost-sharing rules must be simple random-order rules.
\end{abstract}

Key words : cost sharing, additivity, random order 


\section{Introduction}

This paper reconsiders the problem of allocating the cost of a bundle of goods or services among a set of consumers. Numerous examples have been studied in the literature: How should we split the overhead costs of a firm among its various divisions (Shubik (1962))? How should we price the use of public facilities (Loehman and Whinston (1974)), telephone systems (Billera and Heath (1982)), computer networks (Shenker (1995)), transportation systems (Henriet and Moulin (1996)), airport runways (Littlechild and Owen (1973)), irrigation networks (Aadland and Kolpin (1998))? For more examples, we refer the reader to Shubik (1982), Young (1994) and Moulin (1995b).

The bulk of the axiomatic literature on the cost sharing problem has developed in the context of the following model. Agent $i=1, \ldots, n$ demands a quantity $q_{i}$ of a personalized good and the total cost $C\left(q_{1}, \ldots, q_{n}\right)$ must be split equitably among the $n$ agents. The demand vector $q=\left(q_{1}, \ldots, q_{n}\right)$ and the cost function $C$ are the only data of the problem.

Two special cases of this model have received much attention. If $q_{1}=\ldots=$ $q_{n}=1$ and $C$ is defined on $\{0,1\}^{n}$, we obtain the cooperative game model (upon identifying a coalition with the vector in $\{0,1\}^{n}$ whose coordinates corresponding to the members of that coalition are 1 while the others are 0 ). This corresponds to an assumption of complete indivisibility of the goods. In the Aumann-Shapley pricing model, on the other hand, $q_{1}, \ldots, q_{n}$ are arbitrary nonnegative real numbers and $C$ is defined on $\mathbb{R}_{+}^{n}$. Goods are thus assumed to be perfectly divisible. The vast literature on the so-called Aumann-Shapley prices makes that assumption (see, e.g., the survey by Tauman (1988)) and so does most of the recent literature spawn by Moulin and Shenker's (1992) seminal article on the serial mechanism (see, e.g., Friedman and Moulin (1998), Kolpin (1996), Koster et al. (1997), Moulin and Shenker (1994), and Sprumont (1998)).

In a recent paper, Moulin (1995a) proposes a discrete reformulation of the Aumann-Shapley model in which goods come in indivisible units: $q_{1}, \ldots, q_{n}$ are arbitrary nonnegative integers and $C$ is defined over $\mathbb{N}^{n}$. This approach avoids the technical assumptions and complications of the continuous formulation (this turns out to be extremely helpful when addressing the difficult problem of strategic manipulability: see Moulin (1997)) and nicely encompasses the traditional cooperative game model as the special case where all agents demand a single unit.

Our purpose here is to take further advantage of that discrete model to discuss 
informational issues that do not arise in either the cooperative game model or the continuous framework of Aumann and Shapley. Our starting point is the following: trivial observation. In a problem where the demand vector is $q$, the only relevant information is the restriction of the cost function to the vectors between 0 and $q$. In the discrete model, this amounts to $\left(q_{1}+1\right)\left(q_{2}+1\right) \ldots\left(q_{n}+1\right)$ numbers: The relevant information, therefore, gets richer as $q$ grows ${ }^{1}$.

Going one step further, we observe that a cost-sharing problem may be "refined" by adding (or learning) information about costs at "intermediate" production vectors. The simplest refinements obtain by blowing up the measurement scales: instead of knowing costs, say, per ton, we would know them per kilogram. It makes sense for a cost-sharing rule to use such additional information and, perhaps, to revise the cost shares originally decided upon. If the rule is coherent, however, there should exist at least one conceivable refinement of the problem at hand for which the cost shares remain unchanged. This informational coherence property, formally defined in Section 4, is the subject of the paper.

In the tradition of much of the cost-sharing literature, our analysis will be confined to linear cost-sharing rules, i.e., rules that satisfy the straightforward extensions of the additivity and dummy axioms from cooperative game theory to our model. Our main result is Theorem 1 in Section 5: it states that every linear and demand-monotonic rule satisfying (a strict form of) our coherence axiom is a random-order rule in the traditional cooperative game-theoretic sense. (The demand monotonicity property, introduced in Moulin (1995a), merely requires that an agent's cost share does not decrease when his demand increases.) Theorem 1 may be interpreted as an argument for the cooperative game model: even though we have much more information than what is summarized in a cooperative gamebut still less than the "complete" information assumed by the continuous model-, it is difficult to use it in a coherent way.

\section{Model and notations}

The model is essentially that of Moulin (1995a). Let $N=\{1,2, \ldots, n\}, n \geq 2$, be a finite set of agents. Agent $i \in N$ demands good $i$ only; $N$ is therefore also the set of goods. A demand vector is an element $q$ of $\mathbb{N}^{N}$, where $\mathbb{N}=\{0,1,2, \ldots\}$; that is, goods are not perfectly divisible. A cost function is a nondecreasing function $C: \mathbb{N}^{N} \rightarrow \mathbb{R}$ such that $C(0)=0$. The set of all cost functions is denoted by

\footnotetext{
${ }^{1}$ Notice that nothing of the sort happens in the continuous model.
} 
C. A (cost-sharing) problem is a pair $(q ; C)$ in $\mathbb{N}^{N} \times \mathbb{C} . \mathrm{A}$ (cost-sharing) rule $x$ associates with each problem $(q ; C)$ a list of nonnegative cost shares $x(q ; C)$ satisfying the budget balance condition $\sum_{i \in N} x_{i}(q ; C)=C(q)$. The only difference with Moulin's model is that we do not allow for capacity constraints.

It may be worth noting that this formal framework can be given another interpretation than the one suggested by our terminology. One can think of $q_{i}$ as the quantity of some input, perhaps some type of labor, contributed by agent $i$, and reinterpret $C$ as a production function. The function $x$ is then an outputsharing rule. In the sequel, we will stick to the cost-sharing terminology.

Vector inequalities are written $\geq,>, \gg$. If $q$ is a demand vector in $\mathbb{N}^{N}$ and $S \subset N, q_{S}$ denotes the restriction of $q$ to $S$. We sometimes write $q$ as $\left(q_{S}, q_{N-S}\right)$. We also use the notation $-S$ instead of $N-S$ and write $i$ instead of $\{i\}$. If $t \leq q$, the notation $\left[t_{S}, q_{S}\right]$ stands for the integer box $\times_{i \in S}\left\{t_{i}, t_{i}+1, \ldots, q_{i}\right\}$. We write $\left.] t_{S}, q_{S}\right]$ for $\left[t_{S}, q_{S}\right] \backslash\left\{t_{S}\right\}$ and $] t_{S}, q_{S}\left[\right.$ for $\left[t_{S}, q_{S}\right] \backslash\left\{t_{S}, q_{S}\right\}$. A corner of the box $\left[t_{S}, q_{S}\right]$ is a vector in $\times_{i \in S}\left\{t_{i}, q_{i}\right\}$. We denote by $A(q)$ the set of active agents at $q$, i.e., $A(q)=\left\{i \in N: q_{i}>0\right\}$. We let $1^{i}$ stand for the vector in $\mathbb{N}^{N}$ whose $j$ th component is 1 if $j=i$ and 0 otherwise. If $(q ; C)$ is a problem and $q_{i}>0$, we define $\partial_{i} C(q)=C(q)-C\left(q-1^{i}\right)$.

\section{Linearity}

This paper is exclusively concerned with additive rules satisfying the so-called dummy axiom.

Definition 1. A rule $x$ is additive if $x\left(q ; C+C^{\prime}\right)=x(q ; C)+x\left(q ; C^{\prime}\right)$ for every demand vector $q$ and any cost functions $C$ and $C^{\prime}$. It satisfies the dummy axiom if $x_{i}(q ; C)=0$ whenever $\partial_{i} C(t)=0$ for every demand vector $t \in \mathbb{N}^{N}$ such that $t_{i}>0$.

Additive rules satisfying the dummy axiom admit a familiar representation. An agent's cost share is a weighted sum of his marginal costs:

Lemma 1. A rule $x$ is additive and satisfies the dummy axiom if and only if, for every $q \in \mathbb{N}^{N}$ and $i \in A(q)$, there is a mapping $\alpha_{i}^{q}:\left[1^{i}, q\right] \rightarrow \mathbb{R}_{+}$such that

$$
x_{i}(q ; C)=\sum_{t \in\left[1^{i}, q\right]} \alpha_{i}^{q}(t) \partial_{i} C(t) \text { for each } C \in \mathbb{C},
$$

and

$$
\left.\left.\sum_{i \in A(t)} \sum_{s_{-i} \in\left[t_{-i}, q_{-i}\right]} \alpha_{i}^{q}\left(t_{i}, s_{-i}\right)=1 \text { for each } t \in\right] 0, q\right]
$$


This lemma corresponds in our discrete framework to Friedman and Moulin's (1997) representation lemma in the continuous model and to Weber's Theorem 2 in the cooperative game model. A proof of it may be found in Wang (1998). We call the collection $\alpha=\left(\alpha_{i}^{q}\right)_{q \in \mathbb{N}^{N}, i \in A(q)}$ the weight system associated with the rule $x$. This system is unique. Condition (3.2) ensures budget balance and implies that $x_{i}(q ; C)=0$ whenever $q_{i}=0$.

From now on, we adopt the following terminology.

Definition 2. A linear rule is an additive rule satisfying the dummy axiom.

A simple but useful implication of the budget balance condition may be noted right away.

Lemma 2. Let $x$ be a linear rule with associated weight system $\alpha$. Then, for every $q \in \mathbb{N}^{N}$ and every $\left.t \in\right] 0, q[$,

$$
\sum_{i \in A(q): t_{i}>\mathbf{0}} \alpha_{i}^{q}(t)=\sum_{i \in A(q): t_{i}<q_{i}} \alpha_{i}^{q}\left(t+1^{i}\right)
$$

Proof. Let $x$ be a linear rule with associated weight system $\alpha$, let $q \in \mathbb{N}^{N}$ and $t \in] 0, q\left[\right.$. Define the cost functions $C_{t}^{*}$ and $C_{t}^{* *}$ as follows:

$$
\begin{aligned}
C_{t}^{*}(s) & =1 \text { if } s \geq t \text { and } 0 \text { otherwise, } \\
C_{t}^{* *}(s) & =1 \text { if } s>t \text { and } 0 \text { otherwise. }
\end{aligned}
$$

Applying budget balance to $\left(q ; C_{t}^{*}\right)$ and $\left(q ; C_{t}^{* *}\right)$ yields, respectively,

$$
\sum_{i \in A(q): t_{i}>0} \sum_{s_{-i} \in\left[t_{-i}, q_{-i}\right]} \alpha_{i}^{q}\left(t_{i}, s_{-i}\right)=1
$$

and

$$
\sum_{i \in A(q): t_{i}>\mathbf{0}} \sum_{\left.\left.s_{-i} \in\right] t_{-i}, q_{-i}\right]} \alpha_{i}^{q}\left(t_{i}, s_{-i}\right)+\sum_{i \in A(q): t_{i}<q_{i}} \alpha_{i}^{q}\left(t+1^{i}\right)=1 .
$$

Combining these two equations gives (3.3).

It is convenient to introduce at this stage a few examples that will play a role in the sequel. The simplest linear rules are the incremental rules. Let $q \in \mathbb{N}^{N}$ and write $\sum_{i \in N} q_{i}=q(N)$. A path to $q$ is a mapping $\pi^{q}:\{0,1, \ldots, q(N)\} \rightarrow[0, q]$ such that i) $\pi^{q}(0)=0$ and ii) for each $k \in\{0,1, \ldots, q(N)\}, \pi^{q}(k)$ is identical to $\pi^{q}(k-1)$ in all coordinates but one, say, the $i$ th, for which $\pi_{i}^{q}(k)=\pi_{i}^{q}(k-1)+1$. A path system associates with each demand vector $q \in \mathbb{N}^{N}$ a path to $q$. The incremental 
cost-sharing rule generated by a path system $\pi$, or $\pi$-incremental rule, obtains by charging each agent the sum of his marginal costs along the path recommended by the system $\pi$. More precisely, if $(q ; C)$ is a problem and $i \in N$, we compute for every $t_{i} \in\left[1, q_{i}\right]$ the unique integer $k=: k\left(t_{i}\right)$ for which $\pi_{i}^{q}(k-1)=t_{i}-1$ and $\pi_{i}^{q}(k)=t_{i}$, and then charge agent $i$

$$
x_{i}^{\pi^{q}}(q ; C)=\sum_{t_{i} \in\left[1, q_{i}\right]} \partial_{i} C\left(\pi^{q}\left(k\left(t_{i}\right)\right) .^{2}\right.
$$

The best known rules are convex combinations of incremental rules. One possibility is, for each demand vector $q$, to average over all paths to $q$ with identical weight. This was first suggested by Aumann and Shapley (1974).

Definition 3. The Aumann-Shapley rule $x^{A S}$ recommends the arithmetic average of the cost shares computed by the incremental rules: for each problem $(q ; C)$ and $i \in N$,

$$
x_{i}^{A S}(q ; C)=\frac{1}{|P(q)|} \sum_{\pi^{q} \in P(q)} x_{i}^{\pi^{q}}(q ; C),
$$

where $P(q)$ denotes the set of paths to $q$.

An appealing feature of the Aumann-Shapley rule is that it gives positive weight to the marginal costs at all intermediate production vectors. ${ }^{3}$ This implies the nice Converse Dummy axiom: only the dummies pay nothing. Formally,

Converse Dummy. For every problem $(q ; C)$ and $i \in A(q), x_{i}(q ; C)=0$ only if $\partial_{i} C(t)=0$ for every $t \in\left[1^{i}, q\right]$.

One could also concentrate, for each $q$, on those paths that follow the edges of the box $[0, q]$. Let $\Sigma(N)$ be the set of permutations on the agent set $N$. For each $\sigma \in \Sigma(N)$ and $q \in \mathbb{N}^{N}$, denote by $\pi^{q}(\sigma)$ the unique path in $[0, q]$ that assumes the values $\left(q_{S}, 0_{-S}\right), S=\{\sigma(1)\},\{\sigma(1), \sigma(2)\}, \ldots,\{\sigma(1), \sigma(2), \ldots, \sigma(n)\}$.

Definition 4. Let $\lambda: \mathbb{N}^{N} \times \Sigma(N) \rightarrow \mathbb{R}^{N}$ be a weight function assigning to each demand vector $q$ and each permutation $\sigma$ on $N$ a nonnegative weight $\lambda(q, \sigma)$, with $\sum_{\sigma \in \Sigma(N)} \lambda(q, \sigma)=1$ for every $q$. The $\lambda$-random-order rule $x^{\lambda}$ charges to agent $i$ in problem $(q ; C)$ the cost share

$$
x_{i}^{\lambda}(q ; C)=\sum_{\sigma \in \Sigma(N)} \lambda(q, \sigma) x_{i}^{\pi^{q}(\sigma)}(q ; C)
$$

\footnotetext{
${ }^{2}$ This expression should be understood to be zero if $q_{i}=0$.

${ }^{3}$ This property is lost in the continuous model.
} 
If $\lambda$ is constant in $q$, i.e., $\lambda(q, \sigma)=\lambda\left(q^{\prime}, \sigma\right)$ for all $q, q^{\prime}, \sigma$, we call $x^{\lambda}$ a fixedrandom-order rule. The most famous example is the so-called Shapley-Shubik rule $x^{S S}$, where $\lambda(q, \sigma)=1 / n$ ! for all $(q, \sigma)$ : see Shubik (1962). If the demand vector $q$ has an impact only through the set of active agents, i.e., if $\lambda(q, \sigma)=\lambda\left(q^{\prime}, \sigma\right)$ whenever $A(q)=A\left(q^{\prime}\right)$, we call $x^{\lambda}$ a simple random-order rule.

Perhaps the main drawback of the random-order rules is that they violate the converse dummy property. To see how acute a problem this may be, suppose $n=2, q=(1000,1000), C(t)=0$ if $t_{1}+t_{2} \leq 1000$ and $t_{1}<1000$, and $C(t)=1$ otherwise. Although this cost function is almost perfectly symmetric (replacing $C(1000,0)$ with zero would restore symmetry), the Shapley-Shubik rule charges nothing to agent 2 .

\section{Coherence}

We come now to our central axiom, which imposes on the cost-sharing rule a form of coherence with respect to the information available to compute the cost shares. Consider the example of a two-division firm producing, say, beer and soda. The demand for each product is fixed and data on the joint costs of production are available on a per-hectoliter basis. Knowing this cost function, the firm applies a cost-sharing rule to compute each division's cost share. Suppose next that the firm comes to learn its cost function more precisely, say, on a per-liter basis. Sticking to its favorite cost-sharing rule but having more information, it may well decide to modify the original cost shares. However, if the new cost shares differ from the old ones no matter what the refined cost function turns out to be, the rule lacks informational coherence. This is precisely what the axiom that we are about to define rules out.

Let $\mathbb{N}_{+}=\{1,2, \ldots\}$. For every demand vector $q \in \mathbb{N}^{N}$ and every $r=$ $\left(r_{1}, r_{2}, \ldots, r_{n}\right) \in \mathbb{N}_{+}^{N}$, define the vector $r \otimes q:=\left(r_{1} q_{1}, r_{2} q_{2}, \ldots, r_{n} q_{n}\right)$. Say that a cost function $C^{r} r$-refines a cost function $C$ if $C^{r}(r \otimes t)=C(t)$ for every $t \in \mathbb{N}^{N}$.

Coherence. For every problem $(q ; C)$ and every $r \in \mathbb{N}_{+}^{N}$, there exists a cost function $C^{r}$ which $r$-refines $C$ such that $x(q ; C)=x\left(r \otimes q ; C^{r}\right)$.

Let $\Gamma(C ; r)$ be the set of cost functions which $r$-refine $C$. Note that $\Gamma(C ; r)$ is a nonempty convex set, so that its relative interior is nonempty as well. Very much in the spirit of Coherence but slightly stronger is the following condition.

Strict Coherence. For every problem $(q ; C)$ and every $r \in \mathbb{N}_{+}^{N}$, there exists a cost function $C^{r}$ in the relative interior of $\Gamma(C ; r)$ such that $x(q ; C)=x\left(r \otimes q ; C^{r}\right)$. 
Combined with linearity, Coherence turns out to be very demanding. We start off with the following disappointing observation.

Proposition 1. The Aumann-Shapley rule is not coherent.

Proof. Consider the two-agent case $n=2$. The weight system $\alpha$ associated with the Aumann-Shapley rule is given by

$$
\alpha_{i}^{q}(t)=\frac{\left|P\left(t-1^{i}\right)\right||P(q-t)|}{|P(q)|}
$$

for every $q \in \mathbb{N}^{2}, i \in A(q)$, and $t \in\left[1^{i}, q\right]$, where we recall that $P(t)$ is the set of paths from 0 to $t$. In particular, one finds

$$
\alpha_{1}^{(3,2)}(2,1)=\frac{2}{5} \text { and } \alpha_{1}^{(6,2)}(3,1)=\alpha_{1}^{(6,2)}(4,1)=\frac{3}{7} .
$$

Consider the problem $((3,2) ; C)$ given by

$$
C(t)=1 \text { if } t \geq(2,1) \text { or } t_{2} \geq 2 \text {, and } C(t)=0 \text { otherwise. }
$$

Observe that $\partial_{1} C(2,1)=1$ and $\partial_{1} C(t)=0$ if $t \neq(2,1)$. Therefore,

$$
x_{1}^{A S}((3,2) ; C)=\alpha_{1}^{(3,2)}(2,1) \partial_{1} C(2,1)=\frac{2}{5} .
$$

Let $r=(2,1)$. For every cost function $C^{r}$ which $r$-refines $C, \partial_{1} C^{r}(t)=0$ for all $t \notin\{(3,1),(4,1)\}$, and $\partial_{1} C^{r}(3,1)+\partial_{1} C^{r}(4,1)=\partial_{1} C(2,1)=1$. Therefore,

$$
x_{1}^{A S}\left((6,2) ; C^{r}\right)=\alpha_{1}^{(6,2)}(3,1) \partial_{1} C^{r}(3,1)+\alpha_{1}^{(6,2)}(4,1) \partial_{1} C^{r}(4,1)=\frac{3}{7} \neq \frac{2}{5},
$$

contravening Coherence.

\section{Simple random-order rules}

We begin by taking a second look at the simple random-order rules defined in Section 3. Given a demand vector $q$, every cost function $C$ generates a (cooperative) game $^{4} C_{q}$ through the formula

$$
C_{q}(S)=C\left(q_{S}, 0_{N-S}\right) \text { for every } S \subset N
$$

\footnotetext{
${ }^{4}$ That is, a real-valued mapping defined on the set of subsets of $N$ assigning zero to the empty set.
} 
The number $C_{q}(S)$ is just the cost of fully serving the members of $S$, and $C_{q}$ is therefore often called the stand-alone cost game generated by $C$, given $q$. This game is monotonic (i.e., $C_{q}(S) \leq C_{q}(T)$ whenever $S \subset T$ ) and, conversely, given $q$, every monotonic game is the stand-alone cost game generated by some cost function $C$. If $x$ is a simple random-order rule, there is a collection $\left(\xi^{S}\right)_{S \subset N}$ of random-order values ${ }^{5}$ on the set of monotonic games, one for each subset of agents, such that

$$
x(q ; C)=\xi^{A(q)}\left(C_{q}\right) \text { for every problem }(q ; C) .
$$

This means, first, that a simple random-order rule uses very little information to solve any particular cost-sharing problem, namely, the associated stand-alone cost game. But it further means that this information is used in a fairly rigid way: the same random-order value is used for all demand vectors with the same set of active agents.

These features - simplicity and rigidity - guarantee that a simple randomorder rule $x$ is always trivially strictly coherent. In fact, for every problem $(q ; C)$ and $r \in \mathbb{N}_{+}^{N}$, the equality $x(q ; C)=x\left(r \otimes q ; C^{r}\right)$ holds not only for some, but in fact for every, cost function $C^{r}$ which $r$-refines $C$. As we shall see in Section 6 , there are other strictly coherent linear rules. All of them, however, turn out to violate the appealing requirement of Demand Monotonicity introduced by Moulin (1995a):

Demand Monotonicity. For every problem $(q ; C)$ and every agent $i \in N$, $x_{i}(q ; C) \leq x_{i}\left(q+1^{i} ; C\right)$.

Theorem 1. A linear rule satisfies Strict Coherence and Demand Monotonicity if and only if it is a simple random-order rule.

Proof. It is well known (and easily checked) that simple random-order rules are demand-monotonic, and we have just seen that they are strictly coherent.

Conversely, let $x$ be a linear rule with associated weight system $\alpha$. This rule is fixed for the rest of the proof.

Step 1. A consequence of (Strict) Coherence.

Let $q \in \mathbb{N}^{N}, i \in N, r_{i} \in \mathbb{N}_{+}, t \in\left[1^{i}, q\right]$. Denote by $I\left(r_{i} t_{i}\right)$ the integer interval ]$\left.r_{i}\left(t_{i}-1\right), r_{i} t_{i}\right]$ and write $\left(r_{i} q_{i}, q_{-i}\right)=: q^{\prime}$. We claim that if $x$ is coherent, then

$$
\min _{t_{i}^{\prime} \in I\left(r_{i} t_{i}\right)} \alpha_{i}^{q^{\prime}}\left(t_{i}^{\prime}, t_{-i}\right) \leq \alpha_{i}^{q}\left(t_{i}, t_{-i}\right) \leq \max _{t_{i}^{\prime} \in I\left(r_{i} t_{i}\right)} \alpha_{i}^{q^{\prime}}\left(t_{i}^{\prime}, t_{-i}\right)
$$

\footnotetext{
${ }^{5}$ In the usual cooperative game-theoretic sense of Weber (1988, Section 8).
} 
and if $x$ is strictly coherent, both these inequalities must be strict unless $\alpha_{i}^{q^{\prime}}\left(., t_{-i}\right)$ is constant on $I\left(r_{i} t_{i}\right)$.

The argument extends the proof of Proposition 1. Define the cost function $C$ as follows:

$$
C(s)=1 \text { if } s \geq t \text { or } s_{j} \geq t_{j}+1 \text { for some } j \neq i, \text { and } C(s)=0 \text { otherwise. }
$$

Note that $\partial_{i} C(t)=1$ while $\partial_{i} C(s)=0$ whenever $s \neq t$. For any cost function $C^{\prime}$ which $\left(r_{i}, 0_{-i}\right)$-refines $C$, the derivative $\partial_{i} C^{\prime}$ is zero everywhere except possibly at the points $\left(t_{i}^{\prime}, t_{-i}\right), t_{i}^{\prime} \in I\left(r_{i} t_{i}\right)$, and

$$
\sum_{t_{i}^{\prime} \in I\left(r_{i} t_{i}\right)} \partial_{i} C^{\prime}\left(t_{i}^{\prime}, t_{-i}\right)=1
$$

If $\alpha_{i}^{q}\left(t_{i}, t_{-i}\right)<\min _{t_{i}^{\prime} \in I\left(r_{i} t_{i}\right)} \alpha_{i}^{q^{\prime}}\left(t_{i}^{\prime}, t_{-i}\right)$, we must therefore have $x_{i}(q ; C)<x_{i}\left(q^{\prime} ; C^{\prime}\right)$. Likewise, if $\alpha_{i}^{q}\left(t_{i}, t_{-i}\right)>\max _{t_{i}^{\prime} \in I\left(r_{i} t_{i}\right)} \alpha_{i}^{q^{\prime}}\left(t_{i}^{\prime}, t_{-i}\right)$, we obtain $x_{i}(q ; C)>x_{i}\left(q^{\prime} ; C^{\prime}\right)$. This proves (5.1).

To prove the second part of our claim, assume now that, say,

$$
\alpha_{i}^{q}\left(t_{i}, t_{-i}\right)=\min _{t_{i}^{\prime} \in I\left(r_{i} t_{i}\right)} \alpha_{i}^{q^{\prime}}\left(t_{i}^{\prime}, t_{-i}\right)<\max _{t_{i}^{\prime} \in I\left(r_{i} t_{i}\right)} \alpha_{i}^{q^{\prime}}\left(t_{i}^{\prime}, t_{-i}\right)
$$

Observe that a $\left(r_{i}, 0_{-i}\right)$-refinement $C^{\prime}$ of $C$ is completely determined by the values it assumes at the points $\left.\left(t_{i}^{\prime}, t_{-i}\right), t_{i}^{\prime} \in\right] r_{i}\left(t_{i}-1\right), r_{i} t_{i}[$. Calling these values $\gamma_{1}, \ldots, \gamma_{r_{i}-1}$, we may identify the set of $\left(r_{i}, 0_{-i}\right)$ - refinements of $C$ with the set

$$
\Gamma=\left\{\left(\gamma_{1}, \ldots, \gamma_{r_{i}-1}\right): 0 \leq \gamma_{1} \leq \ldots \leq \gamma_{r_{i}-1} \leq 1\right\}
$$

The relative interior of $\Gamma$ obtains by replacing all weak inequalities in the definition of $\Gamma$ with strict inequalities. This relative interior corresponds exactly to the subset of cost functions $C^{\prime}$ for which $\partial_{i} C^{\prime}\left(t_{i}^{\prime}, t_{-i}\right)>0$ for every $t_{i}^{\prime} \in I\left(r_{i} t_{i}\right)$. For such functions, (5.3) implies $x_{i}(q ; C)<x_{i}\left(q^{\prime} ; C^{\prime}\right)$, contravening Strict Coherence. A similar contradiction arises if we assume $\min _{t_{i}^{\prime} \in I\left(r_{i} t_{i}\right)} \alpha_{i}^{q^{\prime}}\left(t_{i}^{\prime}, t_{-i}\right)<$ $\max _{t_{i}^{\prime} \in I\left(r_{i} t_{i}\right)} \alpha_{i}^{q^{\prime}}\left(t_{i}^{\prime}, t_{-i}\right)=\alpha_{i}^{q}\left(t_{i}, t_{-i}\right)$ instead of $(5.3)$.

Step 2. The consequences of Demand Monotonicity.

We claim that if $x$ is demand-monotonic, its weight system $\alpha$ satisfies the following property:

$$
\forall q \in \mathbb{N}^{N}, \forall i \in A(q), \forall t \in\left[1^{i}, q\right], \alpha_{i}^{q+1^{i}}(t)=\alpha_{i}^{q}(t) .
$$


Suppose (5.4) fails: there exist $q \in \mathbb{N}^{N}, i \in A(q)$ and $t \in\left[1^{i}, q\right]$ such that $\alpha_{i}^{q+1^{i}}(t) \neq \alpha_{i}^{q}(t)$. By budget balance, there is no loss in assuming

$$
\alpha_{i}^{q+1^{i}}(t)<\alpha_{i}^{q}(t)
$$

for if the opposite strict inequality holds, there must anyway exist some $t_{-i}^{\prime} \in$ $\left[0, q_{-i}\right]$ such that $\alpha_{i}^{q+1^{i}}\left(t_{-i}^{\prime}, t_{i}\right)<\alpha_{i}^{q}\left(t_{-i}^{\prime}, t_{i}\right)$ in order to ensure that $\sum_{s_{-i} \in\left[\mathbf{0}_{-i}, q_{-i}\right]}$ $\alpha_{i}^{q}\left(t_{i}, s_{-i}\right)=1=\sum_{s_{-i} \in\left[0_{-i}, q_{-i}\right]} \alpha_{i}^{q+1^{i}}\left(t_{i}, s_{-i}\right)$, as is required by $(3.2)$ specialized to the vector $\left(t_{i}, 0_{-i}\right)$. Consider again the cost function $C$ previously defined in $(5.2)$. From (5.5),

$$
x_{i}\left(q+1^{i} ; C\right)=\alpha_{i}^{q+1^{i}}(t)<\alpha_{i}^{q}(t)=x_{i}(q ; C),
$$

violating Demand Monotonicity.

Step 3. Combining the consequences of Strict Coherence and Demand Monotonicity.

Fix $q \in \mathbb{N}^{N}$. From Step 2, we know that for each $i \in A(q)$ there is a mapping $a_{i}: \mathbb{N}_{+} \times\left[0, q_{-i}\right] \rightarrow \mathbb{R}_{+}$such that

$$
\forall q_{i}^{\prime} \in \mathbb{N}_{+}, \forall t \in\left[1^{i},\left(q_{i}^{\prime}, q_{-i}\right)\right], \alpha_{i}^{\left(q_{i}^{\prime}, q_{-i}\right)}(t)=a_{i}(t) .
$$

We claim that for every $t \in[0, q]$, the following is true:

$$
\left\{\begin{array}{c}
\forall i \in A(q), a_{i}\left(., t_{-i}\right) \text { is a constant map and, if } \\
t_{-i} \text { is not a corner of }\left[0, q_{-i}\right], a_{i}\left(., t_{-i}\right) \text { is zero. }
\end{array}\right\}
$$

The proof of this claim is by induction on the number of active agents whose demand is fully met at $t$, namely, $\omega(t):=\left|\left\{i \in A(q): t_{i}=q_{i}\right\}\right|$.

Step 3.1. We prove that (5.7) holds if $\omega(t)=0$.

Fix $t \in[0, q]$ such that $\omega(t)=0$, i.e., $t_{i}<q_{i}$ for all $i \in A(q)$. We use an auxiliary induction argument on the number of active agents whose demand is partially met at $t$, namely, $\delta(t):=\left|\left\{i \in A(q): 0<t_{i}<q_{i}\right\}\right|$.

3.1.1. We start off by showing that (5.7) holds true if $\omega(t)=0$ and $\delta(t)=0$, i.e., $t=0$. In this case (5.7) reduces to the following statement:

$$
\forall i \in A(q), a_{i}\left(., 0_{-i}\right) \text { is a constant map. }
$$


To prove (5.8), note first that each $a_{i}\left(., 0_{-i}\right)$ is nonincreasing:

$$
\forall i \in A(q), \forall s_{i} \in \mathbb{N}_{+}, a_{i}\left(s_{i}, 0_{-i}\right) \geq a_{i}\left(s_{i}+1,0_{-i}\right) .
$$

This is a consequence of (5.6) and budget balance; it follows, e.g., by choosing $q_{i}^{\prime}>s_{i}$ and using $(3.3)$ with $t=\left(s_{i}, 0_{-i}\right)$. Invoking now Coherence, we see that $a_{i}\left(., 0_{-i}\right)$ must be constant on $\mathbb{N}_{+}-\{2\}$ : for if $a_{i}\left(s_{i}, 0_{-i}\right)>a_{i}\left(s_{i}+1,0_{-i}\right)$ for some $s_{i} \geq 2$, then (5.6) and (5.9) would imply, upon choosing $q_{i}^{\prime}>s_{i}$ and noting that $2 s_{i}>s_{i}+1$, that

$$
\forall s_{i}^{\prime} \in I\left(2 s_{i}\right), \alpha_{i}^{\left(q_{i}^{\prime}, q_{-i}\right)}\left(s_{i}, 0_{-i}\right)>\alpha_{i}^{\left(2 q_{i}^{\prime}, q_{-i}\right)}\left(s_{i}^{\prime}, 0_{-i}\right)
$$

According to Step 1, this contradicts Coherence. Strengthening Coherence to Strict Coherence allows us to finally obtain (5.8). For suppose $a_{i}\left(1_{i}, 0_{-i}\right)>$ $a_{i}\left(2_{i}, 0_{-i}\right)$. Choosing $q_{i}^{\prime}>1$, we get

$$
\begin{aligned}
\max _{s_{i}^{\prime} \in\{1,2\}} \alpha_{i}^{\left(2 q_{i}^{\prime}, q_{-i}\right)}\left(s_{i}^{\prime}, 0_{-i}\right) & =\alpha_{i}^{\left(q_{i}^{\prime}, q_{-i}\right)}\left(1_{i}, 0_{-i}\right) \\
& >\alpha_{i}^{\left(q_{i}^{\prime}, q_{-i}\right)}\left(2_{i}, 0_{-i}\right) \\
& =\min _{s_{i}^{\prime} \in\{1,2\}} \alpha_{i}^{\left(2 q_{i}^{\prime}, q_{-i}\right)}\left(s_{i}^{\prime}, 0_{-i}\right)
\end{aligned}
$$

which contradicts the consequence of Strict Coherence established in Step 1.

3.1.2. Having proved (5.8), we now complete the auxiliary induction argument. Fix an integer $d, 0 \leq d<|A(q)|$, and assume that

$$
(5.7) \text { is true whenever } \omega(t)=0 \text { and } \delta(t) \leq d .
$$

We will prove that $(5.7)$ holds for every $t$ such that $\omega(t)=0$ and $\delta(t)=d+1$.

Fix such a $t$. It can be written $t=\left(t_{M}, 0_{N-M}\right)$, where $0_{M} \ll t_{M} \ll q_{M}$ and $M$ has cardinality $d+1$. To prove (5.7), consider first the agents in $M$. For any $i \in M$, construct $t^{\prime}$ by replacing $t_{i}$ with 0 and keeping all other coordinates of $t$ unchanged, i.e., $t^{\prime}=\left(t_{M-i}, 0_{N-(M-i)}\right)$. Since $\omega\left(t^{\prime}\right)=0$ and $\delta\left(t^{\prime}\right)=d$, we may apply the induction hypothesis (5.10) and conclude, since $t_{-i}^{\prime}=t_{-i}$, that

$$
\forall i \in M, a_{i}\left(., t_{-i}\right) \text { is constant, }
$$

and is zero if $t_{-i}$ is not a corner of $\left[0, q_{-i}\right]$. 
Next, apply Lemma 2 to $t$. This yields

$$
\sum_{i \in M} a_{i}(t)=\sum_{i \in A(q)} a_{i}\left(t+1^{i}\right) .
$$

Because of (5.11), this means that $a_{i}\left(t+1^{i}\right)=a_{i}\left(1_{i}, t_{-i}\right)=0$ for every $i \in A(q)-M$. Invoking now the consequence of Strict Coherence derived in Step 1, we conclude that, in fact, the whole map $a_{i}\left(., t_{-i}\right)$ is zero for every $i \in A(q)-M$. This completes our auxiliary induction argument and proves that (5.7) is true whenever $\omega(t)=0$.

Step 3.2. We carry the main induction argument.

Returning to the main induction argument, fix an integer $w, 0 \leq w \leq|A(q)|$, and assume that

$$
\text { (5.7) is true whenever } \omega(t) \leq w \text {. }
$$

We will prove that (5.7) is true for any $t$ such that $\omega(t)=w+1$. Fxactly as in Step 3.1, we use an auxiliary argument by induction on $\delta(t)$.

3.2.1. We start off by showing that (5.7) is true if $\omega(t)=\omega+1$ and $\delta(t)=0$. In this case, we may write $t=\left(0_{M}, q_{N-M}\right)$, where $|(N-M) \cap A(q)|=\omega+1$. In this case, since $t_{-i}$ is a corner of $\left[0, q_{-i}\right]$ for every $i \in A(q),(5.7)$ reduces to the following statement:

$$
\forall i \in A(q), a_{i}\left(., t_{-i}\right) \text { is a constant map. }
$$

To prove (5.13), consider first an arbitrary agent $i \in(N-M) \cap A(q)$. Construct $t^{\prime}$ by replacing $t_{i}=q_{i}$ with $t_{i}^{\prime}<q_{i}$ while keeping all remaining coordinates of $t$ unchanged. Since $\omega\left(t^{\prime}\right)=w$, we may apply the induction hypothesis (5.12) and conclude, since $t_{-i}=t_{-i}^{\prime}$, that

$$
\forall i \in(N-M) \cap A(q), a_{i}\left(., t_{-i}\right) \text { is a constant map, }
$$

To complete the proof, consider next an arbitrary agent $i \in M$. We first show that $a_{i}\left(., t_{-i}\right)$ is nonincreasing, that is to say,

$$
a_{i}\left(s_{i}, t_{-i}\right) \geq a_{i}\left(s_{i}+1, t_{-i}\right)
$$

for every $s_{i} \in \mathbb{N}_{+}$. To see why (5.15) is true, recall (5.6), choose $q_{i}^{\prime}>s_{i}$, and apply (3.3) in Lemma 2 to the vector $\left(s_{i}, t_{-i}\right)=\left(s_{i}, 0_{M-i}, q_{N-M}\right)$ to get

$$
a_{i}\left(s_{i}, t_{-i}\right)+\sum_{j \in(N-M) \cap A(q)} a_{j}\left(s_{i}, t_{-i}\right)=\sum_{j \in M} a_{j}\left(\left(s_{i}, t_{-i}\right)+1^{j}\right) .
$$


Now, for any arbitrary $j \in(N-M) \cap A(q)$, replace $t_{j}=q_{j}$ with $t_{j}^{\prime}<q_{j}$ in the vector $\left(s_{i}, t_{-i}\right)$ and apply the induction hypothesis $(5.12)$ : this yields that $a_{j}\left(s_{i}, t_{-i}\right)=0$. Therefore (5.16) implies (5.15).

Since $a_{i}\left(., t_{-i}\right)$ is nonincreasing, Strict Coherence implies that it is, in fact, constant: the argument is the same as in 3.1 .1 above. Since this is true for every $i \in M,(5.13)$ is proved.

3.2.2. Having established (5.13), we now complete the auxiliary induction argument. Fix $d, 0 \leq d<|A(q)|$, and assume that

$$
\text { (5.7) is true whenever } \omega(t)=w+1 \text { and } \delta(t) \leq d \text {. }
$$

We will prove that (5.7) is true for every $t$ such that $\omega(t)=w+1$ and $\delta(t)=d+1$. Fix such a $t$ and write it $t=\left(\left(t_{L}, 0_{M-L}\right), q_{N-M}\right)$, where $0_{L} \ll t_{L} \ll q_{L},|L|=$ $d+1$, and $|(N-M) \cap A(q)|=w+1$.

Consider first the agents in $(N-M) \cap A(q)$. For any agent $i$ in that set, construct a vector $t^{\prime}$ by replacing $t_{i}=q_{i}$ with some $t_{i}^{\prime}<q_{i}$ and keeping all other coordinates of $t$ unchanged. Note that $\omega\left(t^{\prime}\right)=w$. Applying the induction hypothesis (5.12) yields

$$
\forall i \in(N-M) \cap A(q), a_{i}\left(., t_{-i}\right)=0 .
$$

Next consider any agent $i \in L$. Construct $t^{\prime}$ by replacing $t_{i}>0$ with $t_{i}^{\prime}=0$ and keeping all remaining coordinates of $t$ unchanged. Note that $\delta\left(t^{\prime}\right)=\delta$ while $\omega\left(t^{\prime}\right)=w+1$. Applying the induction hypothesis (5.17) gives us

$$
\forall i \in L, a_{i}\left(., t_{-i}\right) \text { is a constant map }
$$

and it is zero if $t_{-i}$ is a corner of $\left[0, q_{-i}\right]$.

Finally, apply (3.3) in Lemma 2 to $t$. This yields

$$
\sum_{i \in L} a_{i}(t)+\sum_{i \in(N-M) \cap A(q)} a_{i}(t)=\sum_{i \in M} a_{i}\left(t+1^{i}\right),
$$

which, along with (5.18) and (5.19), gives $a_{i}\left(1_{i}, t_{-i}\right)=0$ for every $i \in M-L$. Invoking Strict Coherence and Step 1 again, the latter statement implies that

$$
\forall i \in M-L, a_{i}\left(., t_{-i}\right)=0 .
$$

Gathering (5.18), (5.19), and (5.20), we are done. 
Step 4. Concluding the proof.

Fix $q \in \mathbb{N}^{N}$. Gathering (3.1), (5.6), and (5.7), we get

$$
x_{i}(q ; C)=\sum_{S \subset N-i} \alpha_{i}^{q}\left(q_{S \cup i}, 0_{-(S \cup i)}\right)\left[C\left(q_{S \cup i}, 0_{-(S \cup i)}\right)-C\left(q_{S}, 0_{-S}\right)\right]
$$

for every $i \in A(q)$ and $C \in \mathbb{C}$,where $\alpha_{i}^{q}\left(q_{S \cup i}, 0_{-(S \cup i)}\right) \geq 0$ for every $S \subset N-i$ by $(3.1)$ and $\sum_{S \subset N-i} \alpha_{i}^{q}\left(q_{S \cup i}, 0_{-(S \cup i)}\right)=1$ by budget balance (as can be seen by specializing $(3.2)$ to $\left.t=\left(q_{i}, 0_{-i}\right)\right)$.

This means that, for every $i$, there is an individual probabilistic value (in the sense of Weber (1988, Section 2)) $\xi_{i}^{q}$ defined on the set of monotonic games such that for every cost function $C, x_{i}(q ; C)=\xi_{i}^{q}\left(C_{q}\right)$, where $C_{q}$ is the stand-alone cost game associated with $(q ; C)$. Invoking Theorem 13 in Weber (1988), we conclude that there is a random-order value $\xi^{q}$ on the monotonic games such that $x(q ; C)=\xi^{q}\left(C_{q}\right)$ for all $C \in \mathbb{C}$.

To complete the proof of our Theorem, we must show that $\xi^{q^{\prime}}=\xi^{q}$ whenever $A\left(q^{\prime}\right)=A(q)$. Construct $q^{+}$by replacing all zero coordinates of $q$ with ones, i.e., $q^{+}=\left(q_{A(q)}, 1_{-A(q)}\right)$. For every cost function $C$, define the cost function ${ }^{q^{+}} C$ by

$$
{ }^{q^{+}} C(t)=C\left(q^{+} \otimes t\right) \text { for all } t \in \mathbb{N}^{N}
$$

Thus, $C q^{+}$-refines ${ }^{+} C$ and $q=q^{+} \otimes\left(1_{A(q)}, 0_{-A(q)}\right)$. Since $x$ recommends the same cost shares in all problems that have the same set of active agents and generate the same stand-alone cost game, Coherence implies that $x\left(q ; C^{\prime}\right)=$ $x\left(\left(1_{A(q)}, 0_{-A(q)}\right) ; q^{+} C\right)$ for all cost functions $C^{\prime}$ that $q^{+}$-refine ${ }^{q^{+}} C$. In particular,

$$
x(q ; C)=x\left(\left(1_{A(q)}, 0_{-A(q)}\right) ;^{q^{+}} C\right) .
$$

Returning to (5.21), we conclude that $\alpha^{q}=\alpha^{\left(1_{A(q)}, \mathbf{0}_{-A(q)}\right)}$. Since $q$ is arbitrary, this means that $\alpha^{q^{\prime}}=\alpha^{q}$, hence $\xi^{q^{\prime}}=\xi^{q}$, whenever $A\left(q^{\prime}\right)=A(q)$.

Two corollaries are easily derived from Theorem 1. An unpleasant feature of simple random-order rules which are not fixed-random-order rules is that the cost shares generally depend on the demands for dummy goods. The following axiom rules out such dependence:

Independence of Dummy Demands. For every problem $(q ; C)$ and every agent $i \in N, x(q ; C)=x\left(\left(0_{i}, q_{-i}\right) ; C\right)$ whenever $\partial_{i} C(t)=0$ for all $t \in \mathbb{N}^{N}$ such that $t_{i}>0$. 
Corollary 1. A linear rule satisfies Strict Coherence, Demand Monotonicity, and Independence of Dummy Demands if and only if it is a fixed-random-order rule.

Proof. The "if" statement is clear. To prove the converse, fix a rule $x$ satisfying the four properties assumed in Corollary 1 . By Theorem 1, $x$ is a simple random-order rule with associated weight function $\lambda$. Since this function is constant over all demand vectors with the same set of active agents, we may view it as a mapping on $2^{N} \times \Sigma(N)$. Thus, with a slight abuse of notation, $x(q ; C)=\sum_{\sigma \in \Sigma(N)} \lambda(A(q), \sigma) x_{i}^{\pi^{q}(\sigma)}(q ; C)$ for every problem $(q ; C)$. Consider such a problem. Suppose all agents outside $A(q)$ are dummies, i.e., $\partial_{i} C=0$ for every $i \in N-A(q)$. Writing $\sigma \sim_{A(q)} \sigma^{\prime}$ if $\sigma$ and $\sigma^{\prime}$ rank the agents in $A(q)$ in the same order (i.e., for all $i, j \in A(q), \sigma(i) \leq \sigma(j)$ if and only if $\sigma^{\prime}(i) \leq \sigma^{\prime}(j)$ ), Independence of Dummy Demands implies

$$
\sum_{\sigma^{\prime} \sim A(q)} \lambda\left(A(q), \sigma^{\prime}\right)=\lambda(N, \sigma) \text { for all } \sigma \in \Sigma(N) .
$$

Therefore $x(q ; C)=\sum_{\sigma \in \Sigma(N)} \lambda(N, \sigma) x_{i}^{\pi^{q}(\sigma)}(q ; C)$ for every problem $(q ; C)$, proving the claim.

The second corollary uses the familiar symmetry axiom.

Symmetry. For every problem $(q ; C)$ and any two agents $i, j, x_{i}(q ; C)=x_{j}(q ; C)$ whenever $q_{i}=q_{j}$ and $C$ is symmetric in $i$ and $j$ 's demands.

Corollary 2. The Shapley-Shubik rule is the only linear rule satisfying Strict Coherence, Demand Monotonicity, and Symmetry.

Proof. Use Theorem 1 and the well-known fact that the Shapley value is the only symmetric random-order value.

\section{Discussion}

\subsection{Dropping Demand Monotonicity}

If we do not insist on Demand Monotonicity, we can construct strictly coherent linear rules that are not simple random-order rules. Here is an example for $n=2$ 
and $q \gg 0 .{ }^{6}$ For every $t_{1} \in\left[0, q_{1}\right]$, let $\pi\left(1, t_{1}\right)($.$) be the unique path to q$ whose range contains $\left(t_{1}, 0\right)$ and $\left(t_{1}, q_{2}\right)$. Likewise, for every $t_{2} \in\left[0, q_{2}\right], \pi\left(2, t_{2}\right)($.$) denotes$ the path whose range contains $\left(0, t_{2}\right)$ and $\left(q_{1}, t_{2}\right)$. We refer to such paths as straight paths because, informally speaking, they show no turning point in the interior of the box $[0, q]$. Our rule is a convex combination of the incremental rules generated by the straight paths. We do not weight these paths equally, however. Rather, we attach weight $1 / 2 q_{2}$ to every path $\pi\left(1, t_{1}\right), 0<t_{1}<q_{1}$, and $1 / 2 q_{1}$ to every path $\pi\left(2, t_{2}\right), 0<t_{2}<q_{2}$. The two paths following the edges of the box, namely, $\pi(1,0)=\pi\left(2, q_{2}\right)$ and $\pi\left(1, q_{1}\right)=\pi(2,0)$, receive weight $\left(q_{1}+q_{2}\right) / 4 q_{1} q_{2}$ each.

The weight system (in the sense of Lemma 1 ) associated with this rule is easily computed: for every $q \gg 0$, we obtain

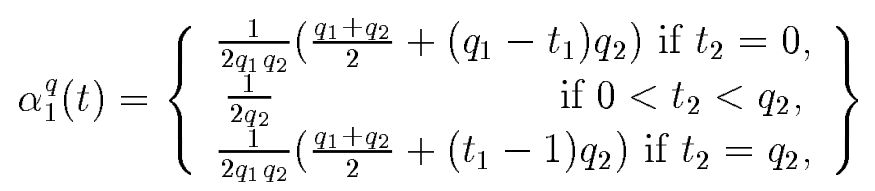

and a completely symmetric expression for $\alpha_{2}^{q}$. Straightforward algebra shows that this weight system satisfies the condition shown to be necessary for Strict Coherence of a linear rule in Step 1 of the proof of Theorem 1. As one easily shows, that condition is also sufficient for Strict Coherence when $n=2$ : our rule is therefore strictly coherent ${ }^{7}$.

The problem of characterizing all the coherent (or strictly coherent) linear rules remains open.

\subsection{Weakening Strict Coherence}

Linear rules that are demand-monotonic and coherent, but not strictly so, need not be random-order rules. An example (which we define again only for $n=2$ and $q \gg 0$ ) is the arithmetic average of the two incremental rules generated by the straight paths $\pi(1,1)$ and $\pi(2,1)$. Note that this rule is symmetric.

We conjecture that the weight system $\alpha$ of any demand-monotonic and coherent linear rule has the property that $\alpha^{q}(t)=0$ whenever $1 \ll t \ll q$.

\footnotetext{
${ }^{6}$ The rule is easily extended to more goods by adopting a simple random-order rule when $n \geq 3$. The assumption that $q \gg 0$ is harmless since the case $A(q)<2$ is trivial when there are only two goods.

${ }^{7}$ Interestingly, the weight system defined by (6.1) is strictly positive, thereby ensuring the Converse Dummy property.
} 


\subsection{Coherence and Scale Invariance}

In the continuous version of the cost-sharing model, Friedman and Moulin (1998) prove that Additivity, Dummy, Scale Invariance, and Demand Monotonicity characterize the simple random-order rules. Scale Invariance is the well-known property that changing the units in which goods are measured does not affect the cost shares.

Perhaps surprisingly, that property has no obvious translation in our discrete framework. For any cost function $C$ and every $r=\left(r_{1}, \ldots, r_{n}\right) \in \mathbb{N}_{+}^{N}$, define ${ }^{r} C(t)=C\left(r_{1} t_{1}, \ldots r_{n} t_{n}\right)$ for every $t \in \mathbb{N}^{N}$. The requirement that

$$
x\left({ }^{r} C ;\left(\frac{q_{1}}{r_{1}}, \ldots, \frac{q_{n}}{r_{n}}\right)\right)=x(C ; q)
$$

is not well defined if some coordinate of the vector $\left(\frac{q_{1}}{r_{1}}, \ldots, \frac{q_{n}}{r_{n}}\right)$ is not integer. On the other hand, the property that

$$
x\left({ }^{r} C ; q\right)=x\left(C ;\left(r_{1} q_{1}, \ldots, r_{n} q_{n}\right)\right)
$$

for all $C, q, r$ is certainly well defined but definitely too strong since it means that every refinement of a given problem should be solved as the original problem.

On the face of it, Coherence bears some resemblance with the continuous Scale Invariance axiom, and our Theorem 1 is reminiscent of Friedman and Moulin's characterization. The correspondence between the two models is far from perfect, however. In particular, it should be kept in mind that the continuous AumannShapley rule is scale-invariant whereas its discrete relative is not coherent.

\section{References}

Aadland, D. and V. Kolpin (1998), "Shared Irrigation Costs: An Empirical and Axiomatic Analysis", Mathematical Social Sciences 35,203-218.

Aumann, R.J. and L. Shapley (1974), Values of Nonatomic Games, Princeton: Princeton University Press.

Billera, L. and D. Heath(1982), "Allocation of Shared Costs: A Set of Axioms Yielding a Unique Procedure", Mathematics of Operations Research 7, 32-39.

Friedman, E. and H. Moulin (1998), "Two Methods to Share Joint Costs or Surplus", forthcoming in Journal of Economic Theory.

Henriet, D. and H. Moulin (1996), "Traffic-Based Cost Allocation in a Network", Rand Journal of Economics 27, 332-345. 
Kolpin, V. (1996), "Multi-Product Serial Cost Sharing: An Incompatibility with the Additivity Axiom", Journal of Economic Theory 69, 227-233.

Koster, M., S. Tijs, and P. Borm (1997), "Serial Cost Sharing for Multi-Commodity Situations", Mathematical Social Sciences, forthcoming.

Littlechild, S. and G. Owen (1973), "A Simple Expression for the Shapley Value in a Special Case", Management Science 20, 370-372.

Loehman, E. and A. Whinston (1974), "An Axiomatic Approach for Cost Allocation of Public Investment", Public Finance Quarterly 2, 236-251.

Moulin, H. (1995a), "On Additive Methods to Share Joint Costs", Japanese Economic Review 46, 303-332.

Moulin, H. (1995b), Cooperative Microeconomics, Princeton: Princeton University Press.

Moulin, H. (1997), "Incremental Cost Sharing: A Characterization by Coalition Strategy-Proofness", mimeo.

Moulin, H. and S. Shenker (1992), "Serial Cost Sharing", Econometrica 60, 10091037 .

Moulin, H. and S. Shenker (1994), "Average Cost Pricing versus Serial Cost Sharing: An Axiomatic Comparison", Journal of Economic Theory 64, 178-201.

Shenker, S. (1995), "Making Greed work in Networks: A Game-Theoretic Analysis of Gateway Service Disciplines", IEEE/ACM Transactions on Networking 3, 819831.

Shubik, M. (1962), "Incentives, Decentralized Control, the Assignment of Joint Costs, and Internal Pricing", Management Science 8, 325-343.

Shubik, M. (1982), Game Theory in the Social Sciences, Cambridge: MTT Press. Sprumont, Y. (1998), "Ordinal Cost Sharing", Journal of Economic Theory 81, 126-162.

Tauman, Y. (1988), "The Aumann-Shapley Prices: A Survey", in A. Roth (ed.), The Shapley Value, Cambridge: Cambridge University Press.

Wang, Y. (1998), "The Additivity and Dummy Axioms in Discrete Cost-Sharing Problems", mimeo.

Weber, R.J. (1988), "Probabilistic Values for Games", in A. Roth (ed.), The Shapley Value, Cambridge: Cambridge University Press.

Young, H.P. (1994), "Cost Allocation", in R. Aumann and S. Hart (eds.), Handbook of Game Theory II, North-Holland. 